\title{
The Impact of IoT in Healthcare: Global Technological Change \& The Roadmap to a Networked Architecture in India
}

\author{
Satya Prakash Dash*
}

\begin{abstract}
The digitization of data including health data (referred to as Internet of Things-loT in Healthcare) and its usage in delivery of healthcare has been growing rapidly across the world. The COVID-19 pandemic has been a pivot for exponential growth of loT in healthcare. Several rapidly evolving technologies are converging to influence the trajectory of IOT in healthcare. There are several challenges in technology development, healthcare delivery as well as issues related to privacy of data, digital divide, role of government and other stakeholders, behaviour and adoption by medical doctors and hospitals. The review article provides an overview of the emergence of IoT in healthcare globally, the intricacies of different factors impinging its current status and recommends policy intervention for an optimal roadmap of IoT in healthcare in the Indian context.
\end{abstract}

Keywords: IOT in healthcare, Digital healthcare, Big data, India, Health, Telemedicine

\section{Introduction: The Advent of New Technologies, Internet Of Things (IoT) and Convergence}

The last two decades have seen extraordinary speeds of technological revolution in the realms of science, including biosciences, and engineering. There has been tremendous progress in the development of molecular biological tools, optical and imaging tools such as tomography, additive and subtractive manufacturing, new fabrication technologies, robotics, sensor technologies, miniaturization of semiconductor chips and of energy storage, increase in computational power and parallel processing, storage of data on semiconductor chips and growth of telecommunication networks. There is also rapid convergence of many of these technologies into what is euphemistically called as the 4th Paradigm of Science ${ }^{1,2}$

In this context of rapid technological change, several parallel factors have led to the advent of what is referred to as Internet of Things (IoT). These include massive improvements in mobile computing and parallel processing powers of hand held devices, improvements in "near field communications" (NFCs) such as Bluetooth ${ }^{\mathrm{TM}}$ low energy technology, evolution of Rapid Frequency Identification (RFID) technology and finally the increase in storage capacity-both on an integrated circuit (chip) as well as in large big data clouds such as that provided by Amazon and Google. The laws of economies of scale including the principles of plentitude have created a seamless integration which has evolved into a network of "connected computing devices or things" across the world that are identifiable by a unique identity. This network is referred to as "Internet of Things" $(\mathrm{IoT})^{3,4}$.

\section{From Lipsticks to Smart Everything}

The term IoT was first coined by Kevin Ashton in 1999. By the late 1990s, the dot com boom was taking place and researchers such as Neil Gershenfeld at Massachusetts Institute of Technology (MIT) were using terms such as "Things That Think" to describe research into the use of sensors on everyday things ${ }^{4-6}$. Kevin Ashton worked
${ }^{1}$ Founding \& Former Head Strategy, Partnerships DBT-BIRAC \&, Member, The Board of Directors Venture Center, Pune, India. *spdash@gmail.com 
at Procter \& Gamble $(P \& G)$ and realized that the bar codes in products provided insufficient information for inventory management. He proposed to $P \& G$ to initiate a research project with MIT to use RFID tags on consumer products such as lipsticks to help track their movement; their usage would ultimately provide market intelligence including inventory management ${ }^{5,6}$. However, it is to be noted that dating the emergence of IoT could be tracked back to John von Neumann's architecture on computing back in $1945^{7}$.

IoT has impacted a multitude of areas and has given rise to what is popularly called "smart everything" including "smart homes", "smart devices \& electronics", "smart automobiles" including driver less cars. IoT's impact on the healthcare industry has increased over the years.

\section{IoT and Healthcare}

The growing trend in healthcare worldwide is preventive, predictive, personalized and participatory and underlying these trends is the increasing digitization of healthcare ${ }^{8}$. IoT in healthcare refers to a network of connected medical devices that are able to not only generate, collect and store data but also connect with a network, analyse the data as well as are able to transmit data of various kinds such as medical images, physiological and vital body signatures and genomics data ${ }^{8-12}$. Increasingly a newer term called Internet of Medical Things (IoMT) to describe connected medtech products is being used. For purposes of this paper, the terms IoT, digital health and IoMT will be used interchangeably.

The IoMT sector is growing at a tremendous pace and is estimated to touch US\$158 billion by 2022 from US $\$ 41$ billion in $2017^{8}$. The recent COVID-19 crisis will hasten the process of hyper growth of this sector.

The IoMT sector has been broadly segmented into the following categories viz ${ }^{8}$.

- Telemedicine and remote consultation

- Consumer healthcare including wearables

- Connected Imaging

- In-patient Monitoring

- Hospital Operations and workflow management

\section{Telemedicine: From NASA's Mercury Program to COVID-19}

The history of telemedicine dates back to the advent of telephony. Lancet in 1879 mentioned the possible use of telephone to reduce the burden of in-person medical consultations ${ }^{13,14}$. Interestingly, the National Aeronautics and Space Administration (NASA) played a crucial role in the further development of telemedicine especially during the Mercury space program and conducted remote health monitoring pilots called the Space Technology Applied to Rural Papago Advanced Health Care (STARPAHC) project ${ }^{13,14}$.

The adoption of telemedicine grew rapidly in the early 2000s as remote monitoring of chronic patients was increased leveraging internet connectivity and video transmission technologies especially mobile telephony and voice over internet protocols (VoIP). Over the past few decades there also have been refinement of data collection protocols especially patient data that has led to development of Electronic Health Records (EHR) that capture the history of patient's medical history including medications, immunizations, symptoms and current diagnosis which is shareable 8,15 .

Telemedicine is an enabler in providing remote care especially in geographically remote areas. It also gained pace in providing home care for senior citizens as well as patients with chronic conditions. As the societal median age of the developed world has shifted towards an older population, the relevance of telemedicine has increased and will continue to increase. Indeed, a new term as "hospital in a home" is gaining ground alluding to the increasing care being provided at home settings remotely. It has also been noticed that use of telemedicine and EHR has improved the quality of General Practitioner-patient consultations ${ }^{8,12,15}$.

The recent COVID-19 pandemic has shown the usefulness of telemedicine consultation in breaking the cycle of infection spread and easy access to consultation. It has been estimated that there has been a ten-fold rise in teleconsultation in the USA in response to the pandemic ${ }^{16}$. The Center for Disease Control and Prevention (CDC) released an advisory guideline for use of telemedicine ${ }^{17}$. This spurt in usage of telemedicine has also been seen in other countries such as China and Italy to name a few ${ }^{16}$. 


\section{Consumer Healthcare and Wearables: Real time health data}

The consumer healthcare or the wearable segment of IoT started gaining pace as consumer wellbeing devices were developed such as Fitbit $^{\mathrm{TM}}$ and Apple Inc.'s smartwatches. These wireless enabled devices could count steps, estimate calories consumed, monitor heartbeat and other cardiac physiology, monitor sleep and provide feedback that is continuous and on-demand. These physiological data could also be transmitted to the cloud and eventually to the care giver for consultation and clinical decisions ${ }^{8,12}$. Many of these features were incorporated into mobile phones as the processing capabilities of mobile phones increased in geometric progression over the last decade. With time, more sophisticated remote monitoring devices were developed such as AliveCor ${ }^{\mathrm{TM}}$ that can measure ECG data and received US Food and Drug Administration (FDA) approval, later Apple Inc. also developed and integrated a similar wearable ECG in its Apple watch- $4^{18}$.

\section{The Digitization of Critical Care and Digital Pathology}

Rapid digitization is taking place in the hospital environment. Many major firms are developing platforms such that multitudes of devices in a hospital can be connected. For example, Philips' Healthsuite, which is an open platform, allows medical devices to share data to a platform which can then process and do analysis of the data that can then be retrieved by healthcare workers including clinicians and nurses (in their mobiles or desktops) amongst others and play a role in informing clinicians' decisions ${ }^{8}$. Philips has also developed an eICU program that combines audio-visual technology along with predictive analytics and data visualization to lay down a centrally monitored intensive care in hospitals (from 50 to 500 ICU beds) through connected devices that provide real time data that helps intensivists and their team provide rapid integrated care across a hospital system ${ }^{19}$.

Another area of IoT is networked data generated and captured from medical devices in critical care settings, diagnosis and monitoring of patients in hospitals such as from CT and MRI scanners, $\mathrm{X}$-ray and mammography devices with integrated HER and imaging outputs that can aid in faster clinical decisions ${ }^{8}$. Digital pathology is the term used to describe actionable information generated using artificial intelligence algorithms on images of diseased tissues including wounds, tumours and other disease conditions ${ }^{8,11}$. Several leading medtech companies such as Philips have brought digital pathology products to the market for example Philips' Intellisite ${ }^{\mathrm{TM}}$ is the first whole slide imaging product that received US FDA approval ${ }^{20}$. In another example that illustrates how medtech firms are combining capabilities, GE and Roche diagnostics have partnered to provide clinical insights to oncologists through development of dashboards that combine GE's healthcare imaging and Roche's capabilities in biomarker, tissue pathology and genomics data and to help clinicians make treatment decisions ${ }^{8}$. Similarly, other global firms such as Siemens have digital pathology products and new medtech companies such as Proscia are building digital pathology technology for oncology.

\section{Making Hospitals Smart-Management of Hospital Assets and Workflows}

Hospitals can be viewed as asset and information intensive organisations. Another use case example of IoT enabled devices and hospital assets such as beds is tracking the utilisation of hospital assets in real time as well as hospital inventory management increasing the efficiency of operations ${ }^{8}$.

Other emerging areas where IoT is starting to create impact is prosthetics, implanted medical devices such as hip joints, defibrillators, nerve stimulators, robotic surgeries to name a few ${ }^{8}$.

\section{Global Challenges and Issues of IoT in Healthcare}

As IoT becomes an indivisible part of healthcare several challenges that cut across geographical lines are emerging. Chiefly they relate to the issues of interoperability, cybersecurity, data privacy and regulation as outlined in sections 9-11.

\section{Interoperability}

Interoperability is one of the biggest challenges faced by the medtech industry as new products are being developed on heterogenous platforms, especially the standards for data collection, storage and transmission used by health systems, including hospital information systems (HIS). Interoperability can be broadly defined as "the ability of two or more systems to exchange information and use the information that has been exchanged" 21 . Interoperability can be further classified into the following ${ }^{21}$.

- technical interoperability that includes data exchange between two systems for e.g. USB to the laptop, 
- syntactic interoperability that specifies format and structure of the data which is specified by standard setting organizations such as Health Level 7 International (HL7) and Fast Healthcare Interoperability Resources (FHIR),

- semantic interoperability that covers the diversity of usage of medical terminologies, ontologies and nomenclatures. There are standard domain specific nomenclatures laboratory observations (Logical Observations Identifiers Names and Codes, LOINC), medicines (Identification of Medicinal Products, IMDP), genes (HUGO Gene Nomenclature Committee, HGNP) and phenotypic abnormalities (Human Phenotype Ontology, HPO) ${ }^{21}$. Navigating interoperability issues will be one of the key issues as IoT in healthcare presence deepens across the world ${ }^{21}$.

\section{Cybersecurity, Data Privacy and Ownership}

Voluminous amount of data especially personalized data is being generated by networked medical devices. This data then is stored in systems and exchanged amongst different stakeholders including healthcare providers (both public and private providers) such as hospitals, payers and insurance companies and researchers 8,10 .

There are concerns about data privacy and security especially since patient identifiable data about health and wellbeing is collected by the IoT enabled devices. One of the underlying policy debates is the ownership of the patient's data and its ethical use as well as the patient's (or individual's) right to erase personal information from a database ${ }^{22,23}$.

The U.S. Department of Health and Human Services (HHS) brought one of the earliest regulations on data management and privacy in 1996 called the The Health Insurance Portability and Accountability Act of 1996 (HIPAA) with privacy and security rules ${ }^{22}$. The HIPAA security rules cover the healthcare providers, insurance companies and clearing houses and aims to protect the privacy of individuals' health information (called protected health information, $\mathrm{PHI}$ ) while being flexible to allow for new technologies to improve the quality of health care and delivery ${ }^{22}$. Additionally, the rules provide procedures for covered entities to assure confidentiality, integrity and availability of $\mathrm{PHI}^{22}$.

Cyberattacks and ransomware are continual risks that health systems face globally. One can imagine how critical care within hospitals especially ICU care, surgeries and many other procedures could get negatively impacted by cyberattacks. In 2017, the WannaCry ransomware attack severely disabled the National Health Service (NHS) in England in which NHS staff were unable to access patient information and data thereby impacting critical services ${ }^{23}$. Similarly, in the USA cybertheft of 80 million health insurance records occurred in $2015^{23}$.

These security concerns have led to US's National Institute of Standards and Technology instituting a cybersecurity framework in 2018 that has taken on-board stakeholders from the US government and private players to collaborate to safeguard and improve healthcare cyber assets across the country ${ }^{23}$.

\section{Medtech Software and Digital Health Regulation: Software as a Medical Device}

While the issues of standards regarding data and cyber security have been mentioned earlier in the article, one of the areas that is rapidly evolving is Medtech device regulation especially in the software domain. As networked devices take centre stage in the digital healthcare space, software and its quality, efficacy and robustness become important since digital healthcare like many other fields are being strengthened to take decisions and these will invariably impact patient's health. The US FDA especially the Center for Devices and Radiological Health (CDRH) has provided the stewardship in regulating medtech products for many decades throughout the product development journey including product registrations $(510 \mathrm{~K})$ and post marketing surveillance ${ }^{24,25}$.

FDA has issued a Digital Health Action Plan outlining its efforts in the digital health sphere ${ }^{24}$. Additionally, it is developing a Digital Health Software Precertification Pilot Program that will facilitate the development of "a more streamlined and efficient regulatory oversight of software based medical devices" ${ }^{\prime 2}$. US FDA has started classifying "Software as a Medical Device" (SAMD) and cites the definition used by International Medical Device Regulators Forum (IMDRF) that states "software intended to be used for one or more medical purposes that perform these purposes without being part of a hardware medical device" ${ }^{24}$. It has also partnered with IMDRF to form a working group on SAMD and has developed quality management systems and a framework of clinical evaluation for SAMD ${ }^{26}$.

As one can imagine, software development is a rapidly evolving field and regulators have to constantly keep pace with changes. For 
example, artificial intelligence (AI) and machine learning (ML) have impacted software development especially in the realm of "adaptive software" which learn from data being generated by the device as well as make clinical decisions such as in digital pathology including detection of cancer and cardiovascular disease. FDA has issued a discussion paper on impact of AI \& ML on software real life modifications and its premarket review ${ }^{27}$.

\section{The Indian Healthcare Scenario}

The advent and integration of Western medicine in India took place during the nineteenth and twentieth century and the architecture of the public health system was inherited from the British era with a tiered approach of tertiary, secondary and primary healthcare system. Over the last seven decades since independence, the healthcare ecosystem in India has grown to be one of the largest sectors in the country.

The market size of Indian healthcare is expected to grow to US $\$ 372$ billion in 2022 from US $\$ 280$ billion in 2020 with more than $74 \%$ in the private health system and out of pocket (OOP) payments ${ }^{28}$.

The public healthcare system is a tiered structure with Sub Centres, Primary Healthcare Centres (PHCs) and Community Health Centres (CHCs). Many PHCs and Sub Centres in rural India are resource starved leading to increasing pressure on $\mathrm{CHCs}$ and tertiary health centres such as All India Institute of Medical Sciences (AIIMS). One also has to be mindful that many areas of India are remote and access still remains a challenge especially in mountainous areas as well as rural areas in the hinterland. While there has been growth in the healthcare infrastructure, which is indeed growing, however, India has historically invested anywhere between 1 and $1.5 \%$ of its GDP on healthcare with current estimates of around $1.6 \%$ (1). There are growing disparities in healthcare delivery between rural and urban India. Health data indicates that there is one doctor available for every 1445 Indians, while the WHO recommendation is for one doctor per every thousand population. Almost 2/3rd of the total number of doctors are located in the urban areas while the rural population in India is $66 \%$ of the total population of India ${ }^{12,29}$.

The disease burden in India reflects the health burdens of the developing world (with high incidences of infectious diseases such as $\mathrm{TB}$ and malaria) as well as the developed world (with growing incidences of chronic diseases such as diabetes, cancer and cardio-vascular diseases). Indicators for maternal and child health, malnutrition, stunting, low birth weight babies have improved but still are a concern.

In the last few years, the Government of India has brought a slew of measures to alleviate the healthcare situation in India. Government has provided its green signal in continuation of the National Health Mission (NHM) and a budget of US $\$ 4.8$ billion has been allocated to NHM in the 2020-21 budget. Other measures such as the Ayushman Bharat-Pradhan Mantri Jan Arogya Yojana which aims to cover 100 million households (500 million Indians) with insurance through subsidized paperless health payments for procedures that are also linked to a Unique Identification number such as Aadhaar.

Health policy makers understand that there is a need for infusion of technology in the healthcare sector to make it more productive, widen the net to increase access and augment the last mile delivery ${ }^{12,29}$.

\section{The Indian Landscape of loT in Healthcare: Building IT Strengths and Regulatory Oversight}

IoT has made rapid strides in India. Telemedicine especially remote monitoring started making its mark in the early 2000s through initiatives by the Indian Space Research Organisation (ISRO) and healthcare providers such as Narayana Hrudalaya that is part of Narayana Health $(\mathrm{NH})$, which runs several hospitals across India. In 2003, NH \& ISRO had partnered to provide tele-consultations in cardiac care which had provided consultations to more than $50 \mathrm{~K}$ patients ${ }^{30}$.

The Information Technology (IT) Act 2000 provided early oversight to the telemedicine sector in India, however it lacked any clear guidelines for data privacy, security and confidentiality. Some initial guidelines were recommended in 2003 by the IT Ministry. Other oversight measures followed such as the Information Technology (Reasonable security practices and procedures and sensitive personal data information) Rules 2011 and the Information Technology (Intermediaries Guidelines) Rules $2011^{31,32}$. Additionally, the Ministry of Health \& Family Welfare (MoH\&FW) notified Electronic Health Records (EHR) standards in 2013 and further revised it in $2016^{33}$. In March 2020, just before India went into a national lockdown for COVID-19, MoH\&FW in partnership with Niti Aayog released guidelines for telemedicine (titled Telemedicine Practice 
Guidelines) that touched upon the behavioral and ethical aspects of consultation between a registered medical practitioner with patients through video, voice, text messaging and social platforms. It outlined the responsibilities for medical practitioner as well as guidelines on e-prescription ${ }^{34}$.

In 2018, Niti Aayog unveiled the National Health Stack that has laid down the architecture of building health IT systems in India. This was further consolidated in the form of the National Digital Health Blueprint (2019) and recently in August 2020, the Government of India launched its National Digital Health Mission $(\mathrm{NDHM})^{35}$.

In the last two decades, the IT infrastructure of India has grown including mobile telephony networks and access to the digital cloud. Several big private hospital chains have digitized their health records and have created EHR systems ${ }^{36}$.

An example of digital transformation in hospital systems is the Narayana Health $(\mathrm{NH})$ group of hospitals that is globally known for providing affordable healthcare at scale. $\mathrm{NH}$ has aimed to develop a home-grown hospital information system (called NH-Atma) over the next few years which will be entirely cloud based and cover the entire NH network. Similarly, NH has developed a data analytics platform called $\mathrm{NH}$ Medha to crunch and extract information which will not only improve hospital's efficiencies but also aid in clinical decisions. $\mathrm{NH}$ has also developed an in-house messaging platform (NH-Kaizala) to facilitate better communication amongst health staff ${ }^{37}$.

$\mathrm{NH}$ has partnered with the government to operationalise e-Health centres that have cloud connectivity integrated with EHR, a unique identification system and diagnostic services. $\mathrm{NH}$ has operationalized nine e-Health centres spread over various states including Karnataka, West Bengal, Rajasthan and Gujarat and, in the year 2016-17, these centres have cumulatively conducted more than 17,000 tele-consultations ${ }^{37}$.

Focusing on screening and alleviating conditions in chronic diseases such as diabetes, $\mathrm{NH}$ instituted an online diabetic management and care system in 2018 and had previously launched another diabetes outpatient application (called CURA in 2016) with the belief that "a diabetologist sitting at home in front of a computer can treat 10 times more patients than seeing patients physically in clinic" and CURA had treated 34,000 patients within two years of its launch ${ }^{37}$. The case study of NH illustrates how a hospital network in
India has primed itself with a stated goal to digitally transform itself.

\section{The Indian Healthcare and Medtech Startups and SMEs \& Their Leap into Digital Health}

Since the beginning of the millennium there has been a growing trend of health technology startups in India and many such as Practo, Lybrate and Mfine have concentrated on digital health mostly focusing on home care and management. These companies have been steadily growing in the fast growing market of home care in India.

In the meantime, over the last two decades, there has been a massive shift in the number of active medtech (devices and diagnostics) startups and SMEs in India through support provided by different arms of the Indian government such as the Technology Development Board (TDB), the then NMITLI program of the Department of Science \& Technology (DST), the Department of Biotechnology (DBT), BIRAC, Millennium Alliance (an initiative supported by the DST, FICCI \& USAID) and the Indo USA Science \& Technology Fund (IUSSTF), Bill \& Melinda Gates Foundation and the Wellcome Trust ${ }^{38-40}$. Together, these programs have created 30004000 startups in India and over $40-50 \%$ are Medtech product development startups ${ }^{38-40}$.

Some Indian startups have predated the funding programs initiated by the Government of India. Startups such as Strand Lifesciences (genomics \& precision medicine), Bigtec/MolBio (molecular diagnostics), Perfint (oncologytumour ablation) have been pioneers of the biotech and medtech startup landscape in India.

Many new startups have taken shape over the last 10 years that are building IoT enabled products for screening, diagnostics and healthcare delivery. These include remote diagnostics and consultation (Neurosynaptic Communications), maternal and child health (CareNx, NemoCare, Sensivision, Janitri, Bempu), AI \& imaging (Predible, Qure.ai; BrainSightAI; Adiuvo Diagnostics; Periwinkle) to chronic disease detection and management such as diabetes (Yostra, BioSense) and wellness to name a few. Table 1 provides an indicative list of Indian Medtech startups and SMEs that are active and many have products that are IoT enabled or they are aiming to make them IoT enabled.

Three brief case studies of IoT in healthcare especially in the Indian scenario are mentioned 


\begin{tabular}{|c|c|}
\hline Area of Medtech & Name of the Indian company \\
\hline Remote Health Monitoring including home visits & $\begin{array}{l}\text { Practo, Lybrate, Mfine, Neurosynaptic, Dozee/Turtle Shell Tech- } \\
\text { nologies, Periwinkle Technologies, Erkadi, Skanray }\end{array}$ \\
\hline Diagnostics including Molecular Diagnostics & $\begin{array}{l}\text { Strand Lifesciences, MolBio, Bigtec, Medgenome, Transasia, J } \\
\text { Mitra \& Co, Robonik, MyLab, Huwel LifeTechnology, Omix, } \\
\text { Achira Labs, PathShodh, Module Innovations, Prantae, } \\
\text { Valetude Primus, VidCare, Affigenix, Healthcube, Exoscan } \\
\text { Heathcare, Fastsense Diagnostics, BioAptagen }\end{array}$ \\
\hline Care \& Critical Care \& monitoring & $\begin{array}{l}\text { Skanray, Erkadi, Trivitron, Sahajanand Medical Technologies, } \\
\text { JyotiCNC, Jeevtronics, Inaccel-Coeo Labs, Atmen Technoven- } \\
\text { tion, Docturnal, Windmill Health, Nocca Robotics, BioDesign } \\
\text { Innovation Labs, Renalyx, Axxonet System Technologies, } \\
\text { Startoon Labs, Monitra, Cardea Biomedicals, Ayu Devices, } \\
\text { Genrich Membranes, CareNx, Nemocare, Janitri, Bempu, } \\
\text { Sattava Medtech, Sensivision, Yostra Labs, Biosense, Cephas, } \\
\text { Dynasense, Agatsa, Pluss Tech/Miracradle, Perfint Health, } \\
\text { Forus, Remidio }\end{array}$ \\
\hline Mental Health & $\begin{array}{l}\text { BrainSightAl, Streben Healthcare, Tick Talk To, PushD, Juno } \\
\text { Clinic, Wysa, Edupysche, InnerHour, }\end{array}$ \\
\hline Al \& Predictive & $\begin{array}{l}\text { Qure Ai, Predible Health, Niramai, Adiuvo Diagnostics, Brain- } \\
\text { SightAl }\end{array}$ \\
\hline Other Technologies including vaccine carriers, drones & $\begin{array}{l}\text { Embryyo Technologies, BlackFrog Technologies, Divish Mobility, } \\
\text { Flytbase Labs, Zipline, Skylark Drones }\end{array}$ \\
\hline
\end{tabular}

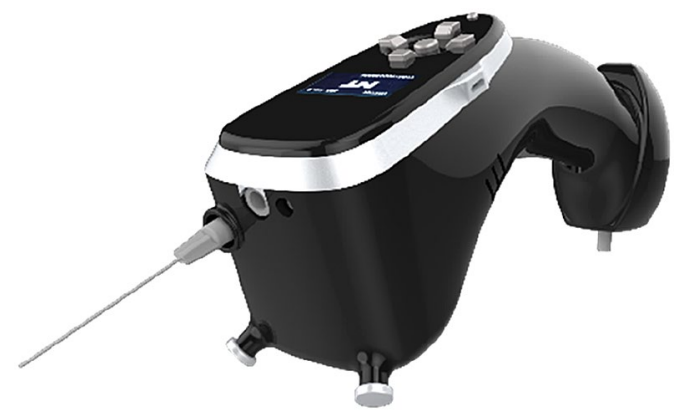

Figure 1: Neuro Touch ${ }^{\mathrm{TM}}$

below. These case studies draw from the experiences of startups in India.

\section{Yostra: Screening and Detection of Peripheral Neuropathy in Diabetic Patients}

India has one of the largest numbers of diabetic patients in the world and most do not have access to diagnostic centres or follow up centres about diabetes related and associated complications. Yostra is a Medtech startup developing products for detection of complications associated with diabetes through its proprietary product, NeuroTouch $^{\mathrm{TM}}$. NeuroTouch ${ }^{\mathrm{TM}}$ is a point of care (Figs. 1, 2), hand-held, battery operated, wireless, screening device for detection of peripheral neuropathy, which is a condition that many diabetics have and the progression of which leads to loss of sense of touch and development of ulcers and wounds that may proceed to gangrene and in many cases amputation of limbs ${ }^{41}$.

Through NeuroTouch ${ }^{\mathrm{TM}}$, a clinician can create a stimulus for patients such as touch (vibrations), temperature (hot \& cold) and pressure (planar pressure for vascular assessment). The responses are then quantified, digitized and stored safely in the cloud. An algorithm then analyses and interprets the data and provides a report to the clinician. The product reduces subjectivity in tests and quantifies the responses of diabetic patient's predisposition to developing peripheral neuropathy and the clinician can then advise for further referrals, which reduces the chances of future complications ${ }^{41}$.

The product has been validated in Manipal University and CMC Vellore India and is currently being used by more than 100 hospitals including leading hospitals in India such as NIMHANS, Bangalore, Military Hospital Hyderabad and the endocrinology department of Narayana Health, Bangalore. Since its deployment in 2019, Neuro Touch ${ }^{\mathrm{TM}}$ has already screened 11,000 patients and clinicians have been informed by the interpreted report ${ }^{41}$. 


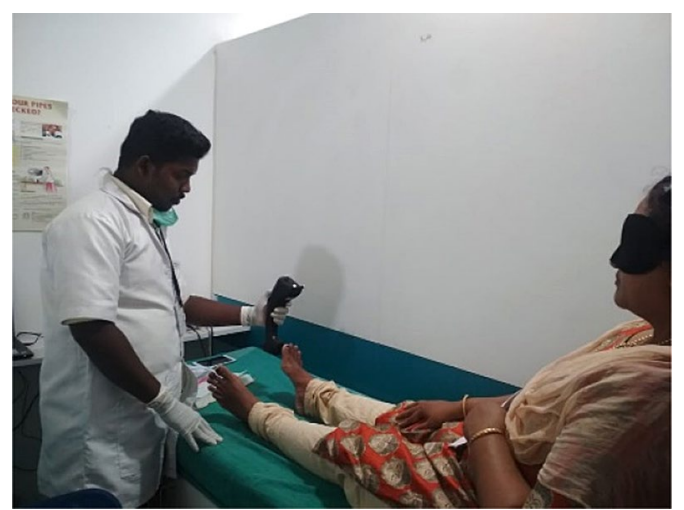

Figure 2: Peripheral Neuropathy screening Neuro Touch ${ }^{\text {TM }}$ (from Vinayak Nandlike, Yostra).

\section{Neurosynaptic's ReMedi-Remote Monitoring in Rural Settings}

As mentioned earlier, India has a relatively poor doctors per unit population ratio. This alludes to the fact that the requirement for teleconsultation is high. Neurosynaptic's is a telemedicine company in India and its ReMedi platform product has solutions to diagnose 20 different diagnostic parameters including ECG, blood pressure, auscultation, oxygen saturation, fetal doppler, blood and urine test. ReMedi, that uses Android based platforms, is powered by lithium polymer rechargeable battery and connects through $3 \mathrm{G}$ or $\mathrm{Wi}-\mathrm{fi}^{42}$.

Over the last 16 years of being operational, ReMedi has operationalized 2500 telehealth centres in rural India and has active projects in 12 states as well as in eight countries in Africa and South East Asia through different implementation partners. This has resulted in providing consultation to 25,000 patients ${ }^{42}$. During the COVID-19 pandemic the need for telemedicine increased and Neurosynaptic received several times more queries about their telemedicine platforms that they have developed indicating the high demand brought by COVID-19. Neurosynaptic developed two more products that were IoT enabled and which provided screening, testing, tracking and reporting of COVID- $19^{42}$.

\section{CareNX: IoT Enabled Solution for Screening for Maternal Care}

CareNx is a medtech startup that is primarily focused on pregnancy care, antenatal care and high-risk pregnancy management. It has built a portfolio of solutions, that are IoT enabled, in pregnancy care such as Homecare, Fetosense (a non-stress test/cardiotocography machine) and AnandiMaa (portable antenatal pregnancy care kit for rural community $)^{43}$.

Fetosense consists of a portable kit with wireless probe which allows remote monitoring with a smart phone and results can also be stored in the cloud. Similarly, AnandiMaa consists of an antenatal care kit consisting of a $\mathrm{Hb}$ meter, a glucometer, a fetal Doppler amongst other devices and the data is smartly analysed by an algorithm and the results then inform a gynaecologist or a healthcare worker about high risk pregnancies and further referrals for the expectant mother for diagnosis and treatment. The portfolio of product helps identify and track high risk pregnancy in hospitals, home, and rural settings (Figs. 3 \& 4) ${ }^{43}$.

CareNx's foot print has expanded to eleven states in India, clocking $100 \mathrm{~K}$ home visits, and the products have screened 35,000 mothers in rural settings (covering 500 villages). The company has collaborated with more than ten NGOs and five hospitals in the last few years of operations ${ }^{43}$.

\section{A Closer Look at National Digital Health Mission (NDHM) and its Founding Document NDHB}

On 15th August 2020, the PM of India announced the launch of NDHM which is founded on the initial vision report by Niti Aayog called National Digital Health Blueprint. The blueprint articulates that it evolved from the Government's (in this case MoH\&FW) policy vision to create a National Digital Health Ecosystem (NDHE) and the word is explicitly pointed out in the blueprint ("an Ecosystem and NOT a system") ${ }^{35}$.

The blueprint further mentions that it is designed as a "layered framework, with its Vision and a set of principles at the core, surrounded by the other layers relating to Digital Health Infrastructure, Digital Health Data Hubs, Building Blocks, Standards and Regulations, and an Institutional Framework for its implementation" $\left({ }^{31}\right)$. The NDHB principles mention universal health coverage (UHC), inclusiveness, security and privacy by design, education and empowerment of citizens, a set of Registries as single sources of truth, open standards, open APIs and a minimalistic approach and further highlights the issues regarding interoperability, data security, privacy and patient safety and data quality ${ }^{35}$. The blueprint is exhaustive and has provided high level guidance for implementation. 

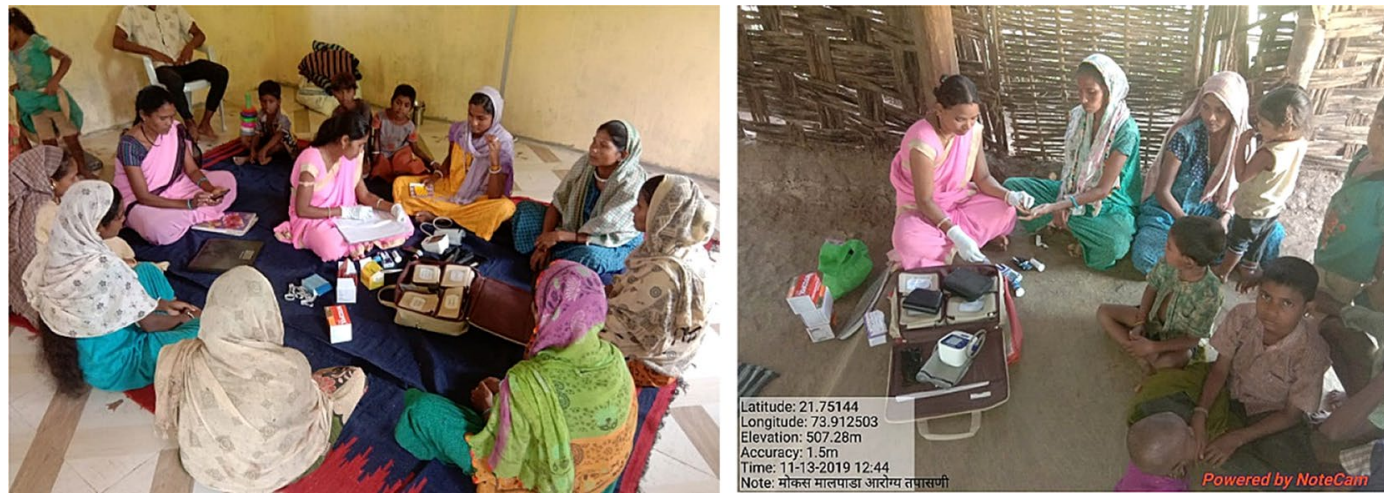

Figures 3 \& 4: CareNx antenatal product being used for health check-up in rural India (from Shantanu Pathak)

NDHM is woven with the patient being at the centre of this designed ecosystem and mentions a personal health identifier or a Health ID (akin to or with likely linkages to Aadhar number- another unique identifier number) which will contain all medical and health related data of the patient including prescriptions, diagnostics reports and other outpatients and in-patients visits to hospital. This data, with patient's consent, will be shareable amongst healthcare providers. NDHM is, to begin with, voluntary, and will be trialed in a few states initially.

Indeed, the scale of NDHM is massive and will usher in a universal health program in India. The concerns about privacy and data protection need to be clarified.

\subsection{Conclusion: Future Gazing The lot In Healthcare In The Indian Scenario \& Its Linkages To Global Trends}

1. COVID-19 as a pivot for India's IoT in Healthcare. Leveraging the opportunity IoT in healthcare (or digital health) will play an increasingly important role in India's healthcare landscape as the country builds and strengthens its digital infrastructure. While telemedicine and remote monitoring were gaining ground in India, the turbulence caused by COVID-19 provided a pivot for digital health to leapfrog. It has been reported that in-person outpatient's traffic fell by $70-80 \%$ and hospitals quickly initiated plans to leverage telemedicine plat- forms. The awareness about digital health increased manifold during the time of pandemic. The latest data from eSanjeevani (The Ministry of Health's tele-consultation platform) shows over $200 \mathrm{~K}$ remote consultations since its launch in November 2019 thus indicating the immense potential for growth in digital healthcare ${ }^{44}$. Since its inception, the e-Sanjeevani platform has been implemented by 23 states across India and cover more than $75 \%$ of the Indian population $^{44}$.

When the pandemic dust settles and our daily lives are recalibrated, digital health will have climbed the ladder of strategic needs of hospitals, big and small across the country. This has the potential to improve healthcare delivery and reduce fraud and leakages in the system to rent seeking agencies. Additionally, it will fuel investments to make organisations including hospitals and healthcare providers to be "digitally ready".

2. Leveraging the Opportunity by technology startups, hospitals and other stakeholdersImproved regulation of IoT enabled Medtech products: the Make in India moment It is evident that the opportunity in front of India is extraordinarily massive and India should leverage this opportunity to be selfreliant or Atmanirbhar as the recent statements by the Government have alluded to. If the digital health implementation is done optimally, it will open massive opportunities for new technology start- 
ups to leverage the infrastructure of the national health stack and build solutions that can truly scale. The innovative ideas in digital health will bring new business models of healthcare delivery alleviating the challenges that India faces in this sector. As the case studies have highlighted, the hospitals, NGOs and other stakeholders are starting to collaborate with new technology startups and this collaboration is set to exponentially grow. It is also important to recognize that many of these "Make in India" products, with high quality and affordability, will have relevance in other geographies such as South East Asia and the African Continent. As Western healthcare is strained, many of the solutions will also have relevance in those markets.

The regulation of medtech devices in India need to integrate the rapid changes occurring in the field especially w.r.t to software as a medical device including adaptive software and the role of artificial intelligence in generating data that informs clinical decisions.

3. Patient's safety and ownership of data at the centre of NDHM

NDHM rightly identifies the patient at the centre of the healthcare ecosystem. NDHB clearly outlines this as well as other areas of importance chiefly inclusiveness. While NDHM is voluntary to begin with, but experience with large mission based Aadhar tells us that it may soon become mandatory. Watertight safeguard measures should be taken and it is critical that the safety and security of patient's data remains paramount. It is already mandated that Indian patient data be stored in local servers; it is crucial that this is implemented.

The blueprint mentions a centralized repository but clarity regarding data ownership should be explicitly stated especially whether it is the patient or the state who has the final ownership of data, the legal consequences of data breach, malware attacks and theft and finally the right of patients to erase healthcare data and the 'right to be forgotten'.

In a country where large swathes still lack digital literacy and understanding of fundamental rights about data and its privacy, it becomes a challenge to raise awareness regarding data privacy. A sustained awareness campaign regarding issues related to data privacy and rights need to be created by the government in partnership with civil society groups.

4. The Digital Equivalence across regions will vary \& the need for public investments in IT \& health-IT infrastructure is high, role of PPP models is important

India faces a digital divide and access to digital infrastructure for its citizen remains highly heterogenous. Therefore, the digital equivalence between regions of the country will vary and this will have implications on adoption of IoT tools for healthcare leading to uneven delivery. Indeed, it is critical that starting from Sub-Centres and PHCs, the IT infrastructure of the healthcare system be strengthened.

India's mobile telephony has grown immensely over the last two decades and the data rates are extremely affordable in comparison to the rest of the world. The mobile telephony user base is set to expand even further and smart phone usage will increase. This bodes well for the growth of IoT or digital tools in healthcare. India also has to invest heavily in the latest of network technology.

5. Behavioural change for adoption of IoT in Healthcare. Developing trust for the new interface between doctors and patients As digital health and IoT tools take off, and a significant portion of consultation and healthcare delivery becomes remote, the behaviour patterns of interaction between healthcare worker or a practitioner with the patient will evolve. It is crucial that trust is built over this interaction and the sociological implication of this needs to be studied.

It is also crucial that the doctors and other healthcare workers are provided continuous training with regards to new technology and new interface that the technology brings. Similarly, patients and lay persons should be made aware to learn about the emerging new technologies and implications of it on their healthcare needs.

6. Skilling the new knowledge workers in IoT in Healthcare and Digital Health

As the demand for IoT in healthcare increases, India will need new skilled human resources and the skill gaps in this arena have to be taken into account. Our pedagogies (in high school, undergraduate and post-graduate institutions) should include IoT in healthcare and the scientific, 
technological, societal and legal issues inherent in this emerging field.

7. Boost in R\&D in IoT in Healthcare is required \& creation of a "Centre for Digital \& Network Society"

India needs to massively invest in $R \& D$ of various areas of IoT, from hardware to software as well as in robotics, artificial intelligence and internet security, digital payment systems, data storage, data encryption technology and data transmission technology. These areas need significant funding both in basic and translational aspects of emerging technologies. It is pertinent to create linkages to industry and funding for startups in this area be boosted. It is suggested that a new "centre for digital \& network society" is established in our topmost scientific institutions such as the Indian Institute of Science with the mandate to study and roadmap new developments in digital technologies that will impact our societies in the future.

8. There is no turning back to the new digital future

As NDHM is implemented, it will show a tantalizing view of the future. The positive outcomes for India especially in the healthcare sector including raising the general health indicators is possible. India can become one of the leading digital societies in the world. The information and the knowledge generated will fuel new innovations in this arena and beyond. There is no turning back to a new future.

\section{Publisher's Note}

Springer Nature remains neutral with regard to jurisdictional claims in published maps and institutional affiliations.

\section{Acknowledgements}

The author would like to thank the following experts Prof. Vijay Chandru, Mr. Krishnakumar Sankaranarayanan, Dr. Malathi Laxmikumaran, Dr. Shyam Vasudev Rao, Mr. Vinayak Nandalike, Mr. Sameer Sawarkar, and Mr. Shantanu Pathak for discussions and valuable inputs.

Received: 27 August 2020 Accepted: 16 September 2020 Published online: 3 November 2020

\section{References}

1. Tony H, Stewart T, Kristin T (2009) The fourth paradigm: data intensive scientific discovery. Published by Microsoft Research. https://www.microsoft.com/en-us/resea rch/wp-content/uploads/2009/10/Fourth_Paradigm.pdf. Accessed 25 Aug 2020

2. Dash SP (2012) Indian biotechnology the roadmap to next decade and beyond ABLE-DBT BIRAC Report. https ://birac.nic.in/webcontent/indian_biotech.pdf. Accessed 25 Aug 2020

3. Clark J (2016) What is internet of things, IBM. https:// www.ibm.com/blogs/internet-of-things/what-is-the-iot/. Accessed 25 Aug 2020

4. M Burgess What is Internet of Things. Wired Explains (2018) https://www.wired.co.uk/article/internet-of-thing s-what-is-explained-iot. Accessed 25 Aug 2020

5. Gabbai A (2019) Kevin aston describes the internet of things. Smithsonian Magazine https://www.smithsonia nmag.com/innovation/kevin-ashton-describes-the-inter net-of-things-180953749/. Accessed 25 Aug 2020

6. Ashton K (2015) Beginnings the internet of things. https ://medium.com/@kevin_ashton/beginning-the-internetof-things-6d5ab6178801. Accessed 25 Aug 2020

7. Firouzi F, Farahani B, Kahng AB, Rabaey JM, Balac N (2017) Guest editorial: alternative computing and machine learning for internet of things. IEEE Trans Very Large Scale Integr (VLSI) Syst 25(10):2685-2687

8. Deloitte, Medtech and Internet of Medical Things (2018) https://www2.deloitte.com/content/dam/Deloitte/global/ Documents/Life-Sciences-Health-Care/gx-lshc-medtechiomt-brochure.pdf. Accessed 25 Aug 2020

9. Gomez-Gonzalez E, Gomez E, Rivas-Marquez J (2020) Artificial intelligence in medicine and healthcare: a review and classification of current and near-future applications and their ethical and social impact. arXiv :2001.09778

10. Canadian Medical Association (2019) The future of technology in health and health care a primer. https:// www.cma.ca/sites/default/files/pdf/health-advocacy/activ ity/2018-08-15-future-technology-health-care-e.pdf. Accessed 25 Aug 2020

11. Niazi MKK, Parwani AV, MN G (2019) Digital pathology and artificial intelligence. Lancet Oncol 20(5):E253-261

12. Bengal Chamber of Commerce (2018) Reimagining the possible in the Indian healthcare ecosystem with emerging technologies, 2018. https://www.pwc.in/assets/pdfs/ publications/2018/reimagining-the-possible-in-the-india n-healthcare-ecosystem-with-emerging-technologies.pdf. Accessed 25 Aug 2020

13. Institute of Medicine (2012) The role of telehealth in an evolving health care environment: workshop summary. The National Academies Press, Washington, DC

14. Nesbitt TS (2017) The evolution of Telehealth, where have we been and where are we going? https://www.ncbi. nlm.nih.gov/books/NBK207141/. Accessed 25 Aug 2020 
15. Zeadally S, Siddiqui F, Baig Z, Ibrahim A (2019) Smart Healthcare Challenges and potential solutions using internet of things (IoT) and big data analytics, PSU Research Review. Emerald Publishing, Bingley

16. Webster P (2020) Virtual Healthcare in era of Covid19. Lancet 395(10231):1180-1181

17. CDC (2020) https://www.cdc.gov/coronavirus/2019ncov/hcp/telehealth.html. Accessed 25 Aug 2020

18. Hendrickson Z (2019) AliveCor drops Kardiaband apple watch accessory-but it isn't losing much. https:// www.businessinsider.com/alivecor-cut-ties-with-apple $-2019-8 ? \mathrm{r}=\mathrm{DE} \& \mathrm{IR}=\mathrm{T}$. Accessed 25 Aug 2020

19. Philips ICU Telemedicine Program (2019) https://www. usa.philips.com/healthcare/resources/landing/teleicu. Accessed 25 Aug 2020

20. Pantanowitz L, Sharma A, Carter AB, Kurc T, Sussman A, Saltz J (2018) Twenty years of digital pathology: an overview of the road travelled, what is on the horizon, and the emergence of vendor-neutral archives. J Pathol Inform 9:40. https://www.ncbi.nlm.nih.gov/pmc/articles/PMC62 89005/. Accessed 25 Aug 2020

21. Lehne M, Sass J, Essenwanger A et al (2019) Why digital medicine depends on interoperability. Dig Med 2:79-83

22. https://www.hhs.gov/hipaa/for-professionals/security/ laws-regulations/index.html. Accessed 25 Aug 2020

23. Ghafur S, Grass E, Jennings NR, Drazi A (2019) The challenges of cybersecurity in healthcare: the UK National Health Service as a case study. Lancet 1:e10-e12

24. USFDA (2020) https://www.fda.gov/medical-devices/ device-advice-comprehensive-regulatory-assistance. Accessed 25 Aug 2020

25. Longitude Prize Sprint Webinar (2020) https://longi tudeprize.org/events/sprint-workshop-1-navigating -medtech-regulation-pathways-developed-and-emerg ing-markets. Accessed 25 Aug 2020

26. https://www.fda.gov/medical-devices/digital-health/softw are-medical-device-samd. Accessed 25 Aug 2020

27. https://www.fda.gov/medical-devices/software-medicaldevice-samd/artificial-intelligence-and-machine-learn ing-software-medical-device. Accessed 25 Aug 2020

28. https://www.ibef.org/industry/healthcare-india.aspx. Accessed 25 Aug 2020

29. https://www.business-standard.com/article/pti-stories/ doctor-patient-ratio-in-india-less-than-who-prescribed -norm-of-1-1000-govt-119111901421_1.html. Accessed 25 Aug 2020

30. https://pharmabiz.com/NewsDetails.aspx?aid=70033 \&sid $=1$. Accessed 25 Aug 2020
31. https://inc42.com/datalab/telemedicine-market-oppor tunity-in-indian-healthtech/https://inc42.com/datalab/ telemedicine-market-opportunity-in-indian-healthtech/. Accessed 25 Aug 2020

32. Nishith Desai Associate, Digital Health in India (2020) https://www.nishithdesai.com/fileadmin/user_uploa d/pdfs/Research_Papers/Digital_Health_in_India.pdf. Accessed 25 Aug 2020

33. Ministry of Health \& Family Welfare, Electronic Health Record Standard for India (2016) https://main.mohfw .gov.in/sites/default/files/17739294021483341357.pdf. Accessed 25 Aug 2020

34. Telemedicine Practice Guidelines (2020) https://www. mohfw.gov.in/pdf/Telemedicine.pdf. Accessed 25 Aug 2020

35. Niti Aayog (2019) National Digital Health Blueprint. https://www.nhp.gov.in/NHPfiles/National_Digit al_Health_Blueprint_Report_comments_invited.pdf. Accessed 25 Aug 2020

36. https://health.economictimes.indiatimes.com/news/ health-it/india-bullish-on-ai-in-healthcare-withoutehr/73118990. Accessed 25 Aug 2020

37. Narayana Health Annual Report, 2018-19 (2019) https ://www.narayanahealth.org/sites/default/files/download/ annual-reports/Annual_Report_2018_19.pdf. Accessed 25 Aug 2020

38. Dash SP (2020) Time to build the Indian medtech industry to global reckoning, Biospectrum. https://www.biosp ectrumindia.com/views/70/16435/time-to-build-india n-medtech-industry-to-global-reckoning.html. Accessed 25 Aug 2020

39. BIRAC 1st BIG Report (2015) https://birac.nic.in/webco ntent/BIRAC_BIG\%2520Report_17\%2520Sept_2015. pdf. Accessed 25 Aug 2020

40. Dash SP, Saberwal G (2018) The bioincubation boom in India. Curr Sci 115(2):228-33. Supplementary Material 1-7. https://www.currentscience.ac.in/Volum es/115/02/0228.pdf. Accessed 25 Aug 2020

41. https://www.neurotouch.co/. Accessed 25 Aug 2020

42. https://neurosynaptic.com/. Accessed 25 Aug 2020

43. https://caremother.in/. Accessed 25 Aug 2020

44. https://health.economictimes.indiatimes.com/news/healt h-it/health-ministrys-esanjeevani-telemedicine-servicerecords-2-lakh-tele-consultations/77635053. Accessed 25 Aug 2020 
Dr Satya Prakash Dash is a technology policy \& strategy professional. He was the founding \& former Head of Strategy, Partnerships and Entrepreneurship Development of DBT-BIRAC (India's nodal biotech innovation agency) where he designed and implemented 15 national biotech \& medtech innovation programs including India's largest early stage life sciences funding program Biotechnology Ignition Grant (BIG), a social innovation program SPARSH \& SIIP (immersion program), an incubation program BioNEST and equity programs BIRAC-SEED \& AcE fund to name a few. He was also the founding co-ordinator for Make in India at
DBT-BIRAC. Previously, he was the Director of Global Innovations at PATH India, $\mathrm{COO}$ at ABLE, Bengaluru, and researcher at University of Cambridge, UK. He is currently Member of Board of Directors at NCL-Venture Center, Pune, India which is one the largest S\&T focused incubator in India with more than 80 resident startups. He has also consulted and advised IIM-Bengaluru, Nesta's Longitude Prize, UK and CARB-X, USA. Dr Dash holds triple masters from the universities of Cambridge UK, Leicester, UK, Sambalpur India and a PhD from University of East Anglia, Norwich UK. 